\title{
Study of Baffle Boundary and System Parameters on Liquid-Solid Coupling Vibration of Rectangular Liquid-Storage Structure
}

\author{
Wei Jing, ${ }^{1}$ Xuansheng Cheng, ${ }^{1,2}$ Wei Shi, ${ }^{1}$ Jin Fan, ${ }^{1}$ and Huan Feng ${ }^{1}$ \\ ${ }^{1}$ School of Civil Engineering, Lanzhou University of Technology, Lanzhou, Gansu 730050, China \\ ${ }^{2}$ Western Center of Disaster Mitigation in Civil Engineering of the Ministry of Education, Lanzhou, Gansu 730050, China \\ Correspondence should be addressed to Xuansheng Cheng; cxs702@126.com
}

Received 10 December 2015; Accepted 16 February 2016

Academic Editor: Salvatore Russo

Copyright (C) 2016 Wei Jing et al. This is an open access article distributed under the Creative Commons Attribution License, which permits unrestricted use, distribution, and reproduction in any medium, provided the original work is properly cited.

In order to study the vibration problem of liquid-solid coupling of rectangular liquid-storage structure with horizontal elastic baffle, ignoring the influence of surface gravity wave, two different velocity potential functions corresponding to the liquid above and below the elastic baffle are assumed; based on the theory of mathematical equation and energy method, the formulas of basic frequency of liquid-solid coupling vibration system are derived, the baffle joined to the tank wall with 3 kinds of boundary conditions, namely, four edges simply supported, two opposite edges clamped and two opposite edges simply supported, and four edges clamped; the influence rules of baffle length-width ratio, the ratio of baffle height to liquid level, baffle elastic modulus, baffle density, baffle thickness, and liquid density on the coupling vibration performance are studied. The results show that the frequency of the clamped boundary is minimum; the influences of baffle length-width ratio and relative height on the basic frequency are much greater than that of the other system parameters; the relation between baffle length-width ratio and the frequency is exponential, while baffle relative height has a parabola relation with the frequency; the larger the baffle length-width ratio, the closer the baffle to the liquid level; the coupling frequency will be reduced more obviously.

\section{Introduction}

Rectangular liquid-storage structure is widely used in water supply and drainage system, sewage treatment, petrochemical industry, TLD of high rise structure, and so on. With the rapid development of our economy, the liquid-storage structures have already started to develop in the direction of large amount and large scale. The special performance of this kind of structure is that it will bear extra dynamic liquid pressure under external excitations.

As a measure to reduce the liquid sloshing, the baffle has been widely used in the field of aerospace, automotive storage tanks, structural engineering, and so on. But setting baffles in the liquid-storage structure, not only does the complexity of the structure itself increase, but also the analysis difficulty is further increased due to the coupling vibration of elastic baffle and liquid [1]; a large amount of researches on the structure with baffle has been carried out all over the world. Wang et al. [2] studied the liquid sloshing characteristics of the spherical tank with baffle, finding that the basic frequency of the spherical tank is decreased after adding baffle. Yue et al. [3] used two potential functions to solve the liquid sloshing problem of the tank with elastic baffle; the results showed that the baffle position in liquid has a great influence on the reducing sloshing effect. Yang et al. [4] carried out finite element simulation calculation and experimental determination of liquid sloshing in a cylindrical liquid-storage tank with an elastic baffle, finding that when the baffle was closer to the liquid surface, its influence on the coupling frequency is greater. Hao et al. [5] used the single Lagrangian method to study stable equilibrium problems of storage tank with different boundary elastic baffle. Biswal and Bhattacharyya [6] considered the dynamic interaction of liquid-structure-baffle system by using the 
finite element method and studied the influences of baffle size and position on the liquid sloshing and structural response. Shahrokhi et al. [7] studied the influences of baffle position on the liquid flow pattern of liquid-storage structure by using computational fluid dynamics (CFD). Wang et al. [8] got the potential functions of each subdomain liquid by using the separation variable method and superposition principle and studied the fluid-solid coupling characteristics of the liquidstorage structure with elastic baffle. Song et al. [9] established a mathematical model of water free surface by using the boundary element method in order to solve the problem of liquid sloshing in a rectangular tank with baffle and pointed out that the reducing sloshing function of baffle is mainly achieved by changing the frequency distribution of original liquid-storage structure. Xue et al. [10] simulated a cube liquid-storage structure with multibaffles and got the conclusion that baffle can effectively reduce the amplitude and dynamic liquid pressure of liquid sloshing. Wang et al. [11] divided the fluid domain into several simple subdomains, and the influences of location, inner radius, and the number of circular baffles on the coupling vibration of the liquid-storage structure were studied. Hasheminejad et al. [12] studied the transient horizontal response of the tank with baffle and the effective baffle shape that can suppress the lateral force. Shekari [13] divided the fluid domain into two parts with baffle, using boundary element method to study the basic mode of liquid-storage structure with baffle, summarizing the maximum seismic responses of the structure with baffle; then the baffle effect on the vibration control of liquidstorage structure is proved. Ebrahimian et al. [14] applied the feature analysis method to study the basic frequency and formation of liquid-storage structure with baffle. Goudarzi and Farshadmanesh [15] studied the free vibration of liquidstorage structure with different size and different position baffle; the results showed that the liquid sloshing height was reduced by $50 \%$ owing to adding baffle.

In summary, although the baffle can exert an inhibitory effect on the liquid sloshing of liquid-storage structure, its effect on the fluid-solid coupling vibration is influenced by many factors. So the effect of elastic horizontal baffle on the fluid-solid coupling vibration of rectangular liquid-storage structure will be studied further in this paper, supposing the liquid is of no rotation, of no viscosity, and incompressible, because the occurrence probability of gravity wave in the structure of civil engineering is small [16]; therefore, the surface gravity wave is ignored. Assuming that the wall of liquid-storage structure is rigid and its baffle is elastic, two different velocity potential functions corresponding to the liquid above and below the elastic baffle are defined; based on the theory of mathematical equation and energy method, the formulas of basic frequency of liquid-solid coupling vibration of rectangular storage structure with flexible baffle are derived, the baffle joined to the tank wall with 3 kinds of boundary conditions, namely, four edges simply supported, two opposite edges clamped and two opposite edges simply supported, and four edges clamped; the conclusions can provide theoretical basis for the reducing sloshing design of reinforced concrete rectangular liquid-storage structure.

\section{Fluid-Solid Coupling Model and Basic Theory}

Assuming that the liquid is in an ideal state, the length, width, and height of the rectangular liquid-storage structure are $a, b$, and $c$, respectively, the distance between elastic baffle and the tank bottom is $H$, liquid level height is $h$, and $h$ is greater than $H$; supposing the wall is rigid, liquid sloshing is of small amplitude. Ignoring hollows in the elastic baffle, although in practical engineering application, in order to make the liquid flow through the baffle, some holes need to be set in the baffle; if the holes are small and arranged in centralized manner, their effect on the coupling vibration can be ignored; when the holes layout does not conform to the above provisions, the calculation results should be modified by considering the liquid flow effect caused by baffle holes [17]; the analytical model is shown in Figure 1.

When the liquid sloshing is of small amplitude, linear potential flow theory can be used to solve the coupling vibration problem. Assuming that the baffle is located below the stationary liquid level, in this case, the velocity potentials for both sides of liquid of the baffle can be expressed as $\phi_{1}(x, y, z, t)$ and $\phi_{2}(x, y, z, t) ; \phi_{1}$ and $\phi_{2}$ all satisfy the Laplace equation [18]:

$$
\nabla^{2} \phi_{j}=0, \quad j=1,2 .
$$

According to the existing literature [19], for common liquids, because $\sigma / \rho_{w}$ is small, for simplicity, liquid surface tension $\sigma$ can be ignored. After neglecting the surface tension of liquid sloshing, the kinematic and dynamic boundary conditions for the first-order liquid sloshing problem can be expressed as follows:

$$
\frac{\partial^{2} \phi_{1}}{\partial t^{2}}+\left.g \frac{\partial \phi_{1}}{\partial z}\right|_{z=h}=0,
$$

where $g$ is the gravity acceleration.

On the contact surface of rigid wall and liquid, the liquid velocity potential functions satisfy the following boundary conditions:

$$
\begin{aligned}
& \left.\frac{\partial \phi_{j}}{\partial x}\right|_{x=0, a}=0 \\
& \left.\frac{\partial \phi_{j}}{\partial y}\right|_{y=0, b}=0 \\
& \quad j=1,2 \\
& \left.\frac{\partial \phi_{2}}{\partial z}\right|_{z=0}=0 .
\end{aligned}
$$

According to Bernoulli equation, the relation between liquid sloshing pressure and velocity potential is $P=$ $-\rho_{w}(\partial \phi / \partial t)$; thus, the total sloshing pressure of the baffle under the action of upper and lower liquid is as follows:

$$
P^{H}=\left(-\rho_{w} \frac{\partial \phi_{1}}{\partial t}\right)-\left(-\rho_{w} \frac{\partial \phi_{2}}{\partial t}\right),
$$




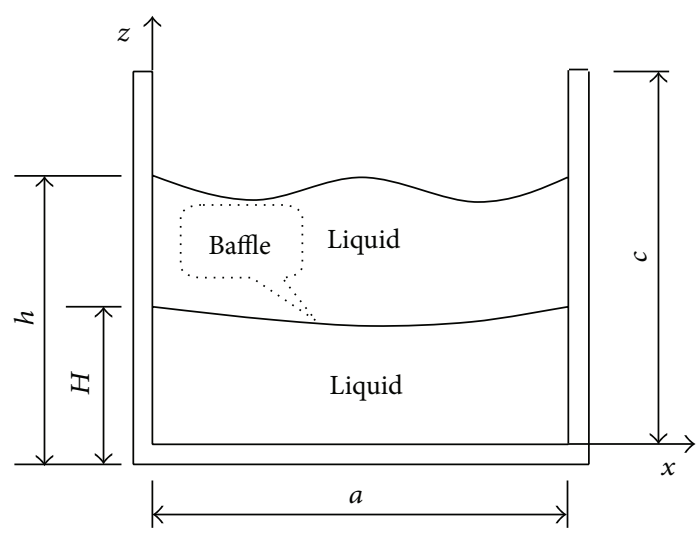

(a) Facade model

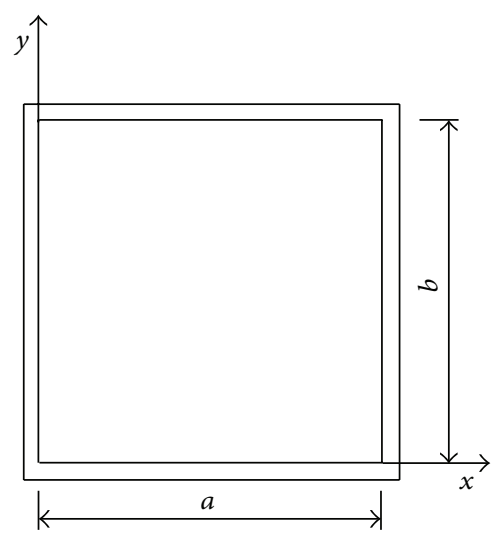

(b) Plane model

Figure 1: Analytical model of liquid-storage structure.

where $\rho_{w}$ is liquid density and $P^{H}$ is total liquid sloshing pressure.

Supposing the deflection in the point $(x, y, H)$ is $W(x, y, t)$, then the differential equation of baffle vibration is

$$
\nabla^{4} W(x, y, t)+\frac{\rho}{D} \frac{\partial^{2} W(x, y, t)}{\partial t^{2}}=-\frac{P^{H}}{D},
$$

where $D$ is the baffle flexural rigidity, $D=E d^{3} /[12(1-\nu)], \rho$ is baffle density, $d$ is baffle thickness, and $v$ is Poisson's ratio.

On the contact surface of liquid and baffle, the velocities in the $z$ direction should be equal to each other; namely,

$$
\frac{\partial \phi_{1}}{\partial z}=\frac{\partial \phi_{2}}{\partial z}=\left.\frac{\partial W}{\partial t}\right|_{z=H}
$$

\section{Coupling Vibration Solution of Liquid-Storage Structure with Baffle}

3.1. Coupled Frequency. Using the method of separation of variables, the velocity potential functions that satisfy (1) and (3) can be expressed as $[3,20,21]$

$$
\begin{aligned}
\phi_{1} & =A_{m n} \cos \left(\frac{m \pi}{a} x\right) \cos \left(\frac{n \pi}{b} y\right)\left(e^{-K_{m n} z}+B_{m n} e^{K_{m n} z}\right) \\
& \cdot e^{i \omega_{m n} t}, \\
\phi_{2} & =C_{m n} \cos \left(\frac{m \pi}{a} x\right) \cos \left(\frac{n \pi}{b} y\right)\left(e^{-K_{m n} z}+D_{m n} e^{K_{m n} z}\right) \\
& \cdot e^{i \omega_{m n} t},
\end{aligned}
$$

where $K_{m n}=\sqrt{(m \pi / a)^{2}+(n \pi / b)^{2}},(m, n=1,2, \ldots) ; \omega_{m n}$ is the basic frequency of the coupling system.
Considering that the liquid sloshing is of small amplitude, in the free liquid surface, $z$ is equal to $h$ approximately; taking (8) into (2) and (4) into (7), we can get

$$
\begin{aligned}
B_{m n} & =\frac{\left(g K_{m n}+\omega_{m n}^{2}\right) e^{-K_{m n} h}}{\left(g K_{m n}-\omega_{m n}^{2}\right) e^{K_{m n} h}}, \\
D_{m n} & =1, \\
C_{m n} & =\frac{A_{m n}\left(B_{m n} e^{K_{m n} H}-e^{-K_{m n} H}\right)}{e^{K_{m n} H}-e^{-K_{m n} H}} .
\end{aligned}
$$

Taking (8), (9), (10), and (11) into (5), the liquid dynamic pressure acting on the baffle can be obtained:

$$
\begin{aligned}
P^{H}= & -\rho i \omega_{m n} \cos \left(\frac{m \pi}{a} x\right) \cos \left(\frac{n \pi}{b}\right) \\
& \cdot A_{m n}\left(e^{-K_{m n} H}+B_{m n} e^{K_{m n} H}\right) \\
& \cdot\left[1-\operatorname{cth}\left(K_{m n} H\right) \frac{B_{m n} e^{K_{m n} H}-e^{-K_{m n} H}}{B_{m n} e^{K_{m n} H}+e^{-K_{m n} H}}\right] e^{i \omega_{m n} t} .
\end{aligned}
$$

Assume that the deflection of elastic baffle is $[20,21]$

$$
W(x, y, t)=W_{m n}(x, y) e^{i \omega_{m n} t} .
$$

By (6), (12), and (13), the following equation can be obtained:

$$
D \nabla^{4} W_{m n}-\rho_{m n} \omega_{m n}^{2} W_{m n}=0,
$$


where $\rho_{m n}$ is a function of $\omega_{m n}$; it can be called dynamic density [21], and

$$
\begin{aligned}
\rho_{m n} & \\
= & \rho \\
& -\frac{\rho_{w}}{K_{m n}}\left[\frac{B_{m n} e^{K_{m n} H}+e^{-K_{m n} H}}{B_{m n} e^{K_{m n} H}-e^{-K_{m n} H}}-\operatorname{cth}\left(K_{m n} H\right)\right] .
\end{aligned}
$$

By (14), the maximum kinetic energy $T_{\max }$ and the potential energy $V_{\max }$ of the elastic baffle can be obtained, respectively:

$$
T_{\max }=\frac{1}{2} \bar{\rho}_{m n} \omega_{m n}^{2} \int_{0}^{a} \int_{0}^{b} W_{m n}^{2}(x, y) d x d y,
$$

$$
\begin{gathered}
V_{\max }=\frac{1}{2} D \int_{0}^{a} \int_{0}^{b}\left\{\left(\frac{\partial^{2} W_{m n}}{\partial x^{2}}+\frac{\partial^{2} W_{m n}}{\partial y^{2}}\right)^{2}-2(1-\nu)\right. \\
\left..\left[\frac{\partial^{2} W_{m n}}{\partial x^{2}} \cdot \frac{\partial^{2} W_{m n}}{\partial y^{2}}-\left(\frac{\partial^{2} W}{\partial x \partial y}\right)^{2}\right]\right\} d x d y .
\end{gathered}
$$

According to the law of energy conservation,

$$
V_{\max }=T_{\max } .
$$

Taking (16) into (17), then

$$
\omega_{m n}^{2} \frac{\rho_{m n}}{D}=d_{m n}
$$

where

$$
d_{m n}=\frac{\int_{0}^{a} \int_{0}^{b}\left\{\left(\partial^{2} W_{m n} / \partial x^{2}+\partial^{2} W_{m n} / \partial x^{2}\right)^{2}-2(1-v) \cdot\left[\left(\partial^{2} W_{m n} / \partial x^{2}\right) \cdot\left(\partial^{2} W_{m n} / \partial x^{2}\right)-\left(\partial^{2} W_{m n} / \partial x \partial y\right)\right]\right\} d x d y}{\int_{0}^{a} \int_{0}^{b} W_{m n}^{2} d x d y}
$$

Taking (9) and $\rho_{m n}$ of (14) into (18),

$$
a_{m n} \omega_{m n}^{4}-b_{m n} \omega_{m n}^{2}+c_{m n}=0
$$

where

$$
\begin{aligned}
a_{m n} & =\left[\rho K_{m n}+\rho_{w} \operatorname{cth}\left(K_{m n} H\right)\right] \operatorname{ch}\left[K_{m n}(h-H)\right] \\
& +\rho_{w} \operatorname{sh}\left[K_{m n}(h-H)\right] ; \\
b_{m n} & =g K_{m n}\left\{\rho K_{m n} \operatorname{sh}\left[K_{m n}(h-H)\right]+\rho_{w} \operatorname{cth}\left(K_{m n} H\right)\right. \\
& \left.\cdot \operatorname{sh}\left[K_{m n}(h-H)\right]+\rho_{w} \operatorname{cth}\left[K_{m n}(h-H)\right]\right\} \\
& +D d_{m n} K_{m n} \operatorname{ch}\left[K_{m n}(h-H)\right] ; \\
c_{m n} & =D d_{m n} K_{m n}^{2} g \operatorname{sh}\left[K_{m n}(h-H)\right] .
\end{aligned}
$$

The basic frequency of the coupling system can be obtained by solving $(20)$ :

$$
\omega_{m n}^{2}=\frac{b_{m n}-\sqrt{b_{m n}^{2}-4 a_{m n} c_{m n}}}{2 a_{m n}} .
$$

As can be seen from (18), in order to obtain the basic frequency of the coupled vibration, the dynamic density $\rho_{m n}$ and $d_{m n}$ must be known firstly; through the above analysis, the liquid dynamic density $\rho_{m n}$ has been obtained; it can be seen that the parameters such as liquid height, horizontal baffle height, and baffle size have a great influence on $\rho_{m n}$. The main factor affecting the expression of $d_{m n}$ of elastic baffle is the boundary conditions. Based on the existing research, the paper derives the expression of $d_{m n}$ of liquidstorage structure with baffle, and the baffle joined to the tank wall with 3 kinds of boundary conditions, namely, four edges simply supported, two opposite edges clamped and two opposite edges simply supported, and four edges clamped; by doing this, the basic frequency of the coupling vibration of the rectangular liquid-storage structure with elastic baffle can be solved.

(1) Four Edges of Baffle Simply Supported. For baffle with four edges simply supported, its boundary conditions are as follows:

$$
\begin{aligned}
& \left.W\right|_{x=0, a}=0, \\
& \left.W\right|_{y=0, b}=0 .
\end{aligned}
$$

Considering the boundary conditions that (23) satisfies, we can set

$$
W_{m n}(x, y)=\sin \frac{m \pi x}{a} \sin \frac{m \pi y}{b} .
$$

Taking (24) into the expression of $d_{m n}$ of (18) gets the following equation:

$$
d_{m n}=\pi^{4}\left(\frac{m^{2}}{a^{2}}+\frac{n^{2}}{b^{2}}\right)^{2} .
$$

(2) Two Opposite Edges of Baffle Clamped and Two Opposite Edges of Baffle Simply Supported. For two opposite edges of baffle clamped and two opposite edges of baffle simply supported, the boundary conditions are as follows:

$$
\begin{aligned}
\left.W\right|_{x=0, a} & =0, \\
\left.\frac{\partial W}{\partial x}\right|_{x=0, a} & =0, \\
\left.W\right|_{y=0, b} & =0 .
\end{aligned}
$$


For two opposite edges of baffle clamped and two opposite edges of baffle simply supported, the deflection equation should satisfy (26); we can suppose

$$
W_{m n}(x, y)=\left(1-\cos \frac{2 m \pi x}{a}\right) \sin \frac{n \pi y}{b} .
$$

Taking (27) into the expression of $d_{m n}$ of (18), we can get

$$
d_{m n}=\frac{1}{3}\left(\frac{2 m \pi}{a}\right)^{4}+\left(\frac{n \pi}{b}\right)^{4}+\frac{2}{3}\left(\frac{2 m \pi}{a}\right)^{2}\left(\frac{n \pi}{b}\right)^{2} \text {. }
$$

(3) Four Edges of Baffle Clamped. For baffle with four clamped edges, it has the following boundary conditions:

$$
\begin{gathered}
\left.W\right|_{x=0, a}=0, \\
\left.\frac{\partial W}{\partial x}\right|_{x=0, a}=0, \\
\left.W\right|_{y=0, b}=0, \\
\left.\frac{\partial W}{\partial x}\right|_{y=0, b}=0 .
\end{gathered}
$$

Considering boundary conditions equations (29) for a rectangular baffle with four clamped edges, the baffle deflection equation can be expressed as

$$
W_{m n}(x, y)=\left(1-\cos \frac{2 m \pi x}{a}\right)\left(1-\cos \frac{2 m \pi y}{b}\right) \text {. }
$$

Taking (30) into the expression of $d_{m n}$ of (18), then

$$
\begin{aligned}
& d_{m n} \\
& =\frac{1}{3}\left[\left(\frac{2 m \pi}{a}\right)^{4}+\left(\frac{2 n \pi}{b}\right)^{4}+\frac{2}{3}\left(\frac{2 m \pi}{a}\right)^{2}\left(\frac{2 n \pi}{b}\right)^{2}\right] .
\end{aligned}
$$

Through the above derivation, as can be seen from (20) and (22), the main factors affecting the basic frequency of the coupling vibration of rectangular liquid-storage structure with baffle include liquid density, baffle density, baffle thickness, the ratio of baffle height to liquid level, the ratio of baffle length to width, and baffle boundary conditions. For convenience of engineering application, supposing the ratio of baffle length to width is $\lambda=b / a$ and the ratio of baffle height to liquid level is $\gamma=H / h$ and taking $m=n=1$, then the fundamental frequency of the coupling system can be gotten by (22):

$$
\omega_{11}^{2}=\frac{b_{11}-\sqrt{b_{11}^{2}-4 a_{11} c_{11}}}{2 a_{11}},
$$

where

$$
\begin{aligned}
& b_{11}=g K_{11}\left\{\rho K_{11} \operatorname{sh}\left[K_{11}(h-H)\right]+\rho_{w} \operatorname{cth}\left(K_{11} H\right)\right. \\
& \left.\cdot \operatorname{sh}\left[K_{11}(h-H)\right]+\rho_{w} \operatorname{cth}\left[K_{11}(h-H)\right]\right\} \\
& +D d_{11} K_{11} \operatorname{ch}\left[K_{11}(h-H)\right] ; \\
& c_{11}=D d_{11} K_{11}^{2} g \operatorname{sh}\left[K_{11}(h-H)\right] ; \\
& K_{11}=\frac{\pi}{a} \sqrt{1+\frac{1}{\lambda^{2}}} .
\end{aligned}
$$

3.2. Theory of Coupled Modes. In order to solve the coupled modes corresponding to the basic coupled frequency of liquid-storage structure with horizontal elastic baffle, we can assume (34) based on (8):

$$
\phi_{j}=\Phi_{j}(x, y, z) e^{i \omega_{m n} t} ; \quad(j=1,2) .
$$

Taking (34) into continuity equation (1) of liquid sloshing in the fluid domain $V$, (2) of kinematic and dynamic boundary conditions of liquid free surface $\partial S_{f}$, boundary condition equations (3) and (4) in the liquid-solid interaction wall $\partial S_{w}$, and same speed condition equation (7) in the baffle $(z=H)$, respectively, then the differential boundary value equation of characteristic mode function can be obtained:

$$
\begin{gathered}
\nabla^{2} \Phi_{j}=0, \quad(j=1,2) ; \quad V, \\
\frac{\partial \Phi_{1}}{\partial z}=\frac{\omega_{m n}^{2}}{g} \Phi_{1} ; \quad \partial S_{f}, \\
\frac{\partial \Phi_{j}}{\partial x}=\frac{\partial \Phi_{j}}{\partial y}=\frac{\partial \Phi_{2}}{\partial z}=0 ; \quad \partial S_{w}, \\
\frac{\partial \Phi_{1}}{\partial z}=\frac{\partial \Phi_{2}}{\partial z}=\frac{\partial W}{\partial t}=0 ; \quad z=H .
\end{gathered}
$$

Equation (35) can be solved by FEM method, and the equation should be firstly transformed into functional extremes problem:

$$
\delta L(\Phi)=0,
$$

where $L$ is the functional; its expression is [22]

$$
\begin{aligned}
L= & \int_{V}\left[\left(\frac{\partial \Phi}{\partial x}\right)^{2}+\left(\frac{\partial \Phi}{\partial y}\right)^{2}+\left(\frac{\partial \Phi}{\partial z}\right)^{2}\right] d V \\
& -\frac{\omega_{m n}^{2}}{g} \int_{\partial S_{f}} \Phi_{j}^{2} d S .
\end{aligned}
$$

After the finite element discretization of the liquid, $\Phi$ of each element $V_{e}$ can be obtained by interpolation method [23]:

$$
\Phi(x, y, z)=\sum_{k=1} N_{k} \Phi_{k}^{e}=\mathbf{N}^{T} \Phi_{e}
$$

where $\mathbf{N}=\left(N_{1}, N_{2}, \ldots, N_{j}\right)^{T}$ is shape function array in the fluid domain and $\Phi_{e}=\left(\Phi_{e 1}, \Phi_{e 2}, \ldots, \Phi_{e k}\right)$ is the corresponding node array.

Similarly, $\Phi$ of each element $S_{f e}$ in the free surface can be expressed as

$$
\Phi(x, y, z)=\sum_{l=1} \widetilde{N}_{l} \Phi_{l}^{e}=\widetilde{\mathbf{N}}^{T} \Phi_{e}
$$

where $\widetilde{\mathbf{N}}=\left(\widetilde{N}_{1}, \widetilde{N}_{2}, \ldots, \widetilde{N}_{l}\right)$ is shape function array in the free surface and $\Phi_{e}=\left(\Phi_{e 1}, \Phi_{e 2}, \ldots, \Phi_{e l}\right)$ is the corresponding node array.

Taking (38) and (39) into $L$

$$
\begin{aligned}
L & =\sum \boldsymbol{\Phi}_{e}^{T} \mathbf{C}_{e} \Phi_{e}-\frac{\omega_{m n}^{2}}{g} \sum \boldsymbol{\Phi}_{e}^{T} \mathbf{D}_{e} \boldsymbol{\Phi}_{e} \\
& =\boldsymbol{\Phi}^{T} \mathbf{C} \boldsymbol{\Phi}-\frac{\omega_{m n}^{2}}{g} \boldsymbol{\Phi}^{T} \mathbf{D} \boldsymbol{\Phi},
\end{aligned}
$$


where $\mathbf{C}$ and $\mathbf{D}$ are formed by element matrixes $\mathbf{C}_{e}$ and $\mathbf{D}_{e}, \boldsymbol{\Phi}$ is integrated node variable array, and

$$
\begin{aligned}
& \mathbf{C}_{e}=\sum_{V_{e}}\left(\frac{\partial \mathbf{N}}{\partial x} \frac{\partial \mathbf{N}^{T}}{\partial x}+\frac{\partial \mathbf{N}}{\partial y} \frac{\partial \mathbf{N}^{T}}{\partial y}+\frac{\partial \mathbf{N}}{\partial z} \frac{\partial \mathbf{N}^{T}}{\partial z}\right) d V \\
& \mathbf{D}_{e}=\int_{S_{f e}} \widetilde{\mathbf{N}} \widetilde{\mathbf{N}}^{T} d S_{f} .
\end{aligned}
$$

Taking (40) into (36),

$$
\mathbf{C} \Phi-\frac{\omega_{m n}^{2}}{g} \mathbf{D} \Phi=0 .
$$

\section{Numerical Examples and Discussions}

According to (32), the basic frequency of the liquid-solid coupling vibration of rectangular liquid-storage structure with horizontal baffle can be solved, in order to study the coupling vibration characteristics of solid-liquid coupling system more comprehensively, assuming the baffle joined to the tank wall with 3 kinds of boundary conditions, taking a variety of values for the main system parameters of this kind of structure; by doing this, statistical results can be obtained. The liquid height $h$ is $3 \mathrm{~m}$, the baffle width is $4 \mathrm{~m}$, and the other parameters are as follows.

4.1. Verification of Proposed Method. Equation (22) is the basic frequency of three-dimensional coupling system considering the elasticity of horizontal baffle, when the baffle is assumed to be rigid; namely, baffle bending stiffness $D$ tends to infinity, the fluid-solid coupling vibration in the upper baffle can be approximately equivalent to liquidstorage structure without baffle, and the liquid coupling frequency above the rigid baffle with four clamped edges can be obtained by (22):

$$
\begin{aligned}
& \omega_{m n} \\
& =\sqrt{g \sqrt{\left(\frac{m \pi}{a}\right)^{2}+\left(\frac{n \pi}{b}\right)^{2}} \cdot \operatorname{th}\left[\sqrt{\left(\frac{m \pi}{a}\right)^{2}+\left(\frac{n \pi}{b}\right)^{2}} \cdot(h-H)\right]} .
\end{aligned}
$$

For the two-dimensional rectangular liquid-storage structure, the coupling frequency can be expressed as [23]

$$
\omega_{n}=\sqrt{\frac{g n \pi}{b} \operatorname{th}\left(\frac{n \pi(h-H)}{b}\right)} .
$$

By comparing (43) and (44) of basic coupling frequency in the three- and two-dimensional coupling vibration problem, the calculation method rationality of the coupling frequency is explained to a certain extent.

On the other hand, in order to further verify the method rationality of the coupling vibration of rectangular liquidstorage structure with different boundary baffle, the corresponding calculation model is established by ADINA. Baffle length to width ratio $\lambda$ is 1.0 , baffle height-liquid level ratio $\gamma$ is $0.1,0.3,0.5,0.7$, and 0.9 , respectively, baffle thickness $d$

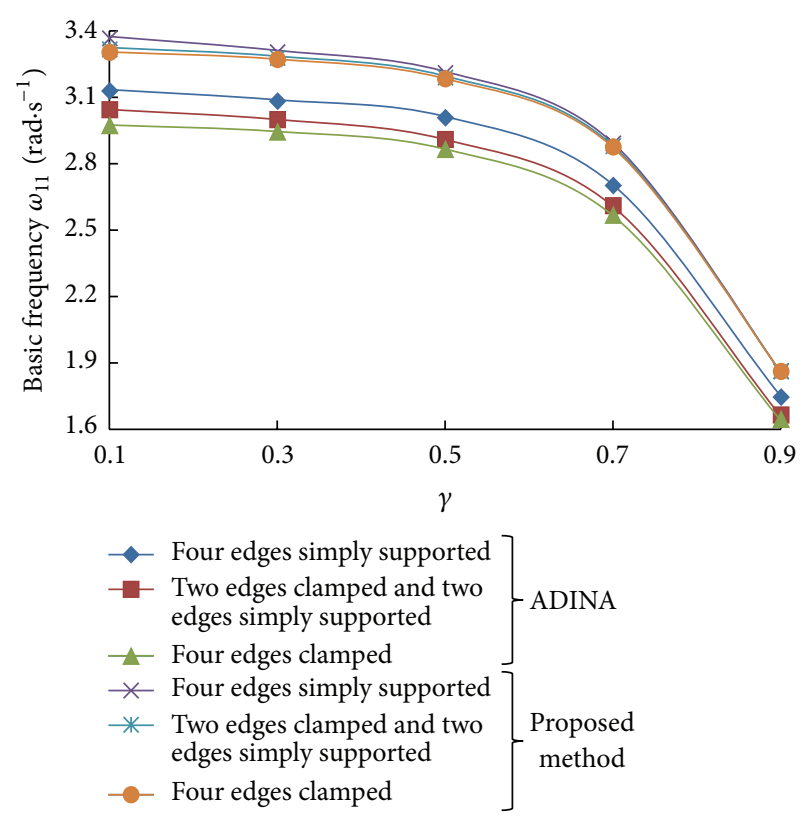

FIgURE 2: Results comparison.

is $6 \mathrm{~cm}$, baffle elastic modulus $E$ is $30 \mathrm{GPa}$, baffle density $\rho$ is $2500 \mathrm{~kg} / \mathrm{m}^{3}$, and $\rho_{w}$ is $1000 \mathrm{~kg} / \mathrm{m}^{3}$. Comparison of calculation results of ADINA and (32) is shown in Figure 2.

As seen from Figure 2, the difference of coupling frequency results calculated by the proposed method and ADINA is small; besides, with the change of $\gamma$, change trends of frequency corresponding to the two methods are consistent; then the validity of the present method is verified.

\subsection{Analysis of Boundary and Parameter Influence}

4.2.1. Effect of Baffle Length-Width Ratio on the Basic Frequency of Liquid-Solid Coupling Vibration. The ratios of baffle length to width $\lambda(\lambda=b / a)$ are, respectively, 1.0, 1.5, 2.0, 2.5 , and 3.0, baffle thickness $d$ is $6 \mathrm{~cm}$, the ratio of baffle height to liquid level $\gamma(\gamma=H / h)$ is 0.5 , liquid density $\rho_{w}$ is $1000 \mathrm{~kg} / \mathrm{m}^{3}$, baffle density $\rho$ is $2500 \mathrm{~kg} / \mathrm{m}^{3}$, baffle elastic modulus $E$ is $30 \mathrm{GPa}$, and the influence of $\lambda$ on the basic frequency of the coupling vibration is shown in Figure 3. As can be seen from Figure 3, the natural frequencies of the coupling system under the three boundary conditions are decreased with the increase of baffle length-width ratio, and the relationship between length-width ratio and the natural frequency is exponential. Under the same lengthwidth ratio, natural frequency corresponding to four edges of baffle simply supported is maximum, natural frequency corresponding to two opposite edges simply supported and two opposite edges clamped is middle, and natural frequency corresponding to four edges clamped is minimum; namely, the basic frequency of the coupling system decreases with the strengthening of the baffle constraint. Therefore, horizontal baffle should be connected to tank wall with four clamped edges when conducting sloshing reduction design; it not only 


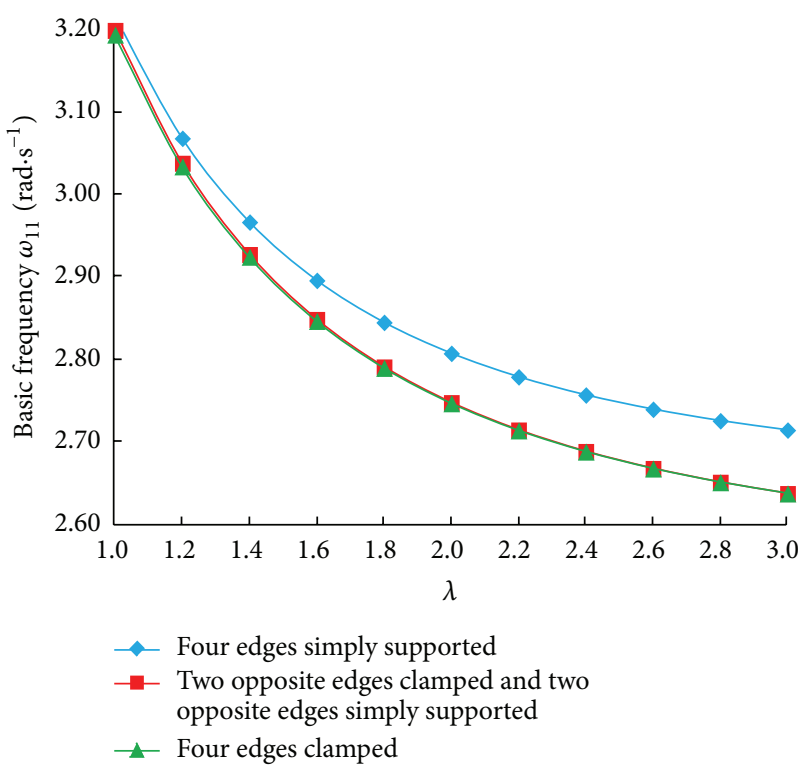

FIGURE 3: Effect of baffle length-width ratio $\lambda$ on the basic frequency of liquid-solid coupling vibration.

is convenient for construction, but also can reduce the basic frequency of fluid-solid coupling vibration.

\subsubsection{Effect of Baffle Height-Liquid Level Ratio on the Basic} Frequency of Liquid-Solid Coupling Vibration. The ratios of baffle height to liquid level $\gamma(\gamma=H / h)$ are, respectively, $0.1,0.2,0.3,0.4,0.5,0.7,0.8$, and 0.9 , baffle length-width ratio $\lambda$ is 1.0 , baffle thickness $d$ is $6 \mathrm{~cm}$, liquid density $\rho_{w}$ is $1000 \mathrm{~kg} / \mathrm{m}^{3}$, baffle density $\rho$ is $2500 \mathrm{~kg} / \mathrm{m}^{3}$, baffle elastic modulus $E$ is $30 \mathrm{GPa}$, and the influence of $\gamma$ on the basic frequency of the coupling vibration is shown in Figure 4. Figure 4 shows that the basic frequency of the coupling vibration under the three boundary conditions decreases with the increase of baffle height to liquid level ratio; when the ratio $\gamma$ changes in the range of $0.1 \sim 0.5$, the basic frequency reduces relatively slow, but when $\gamma$ changes from 0.5 to 0.9 , that is, the baffle is closer to the liquid surface, the trend that frequency decreases is much faster; therefore, in the actual project, baffle should be designed as far as possible near to the liquid surface. When the ratio $\gamma$ is smaller, the basic frequency corresponding to the three boundary conditions has a certain difference; namely, basic frequency corresponding to four edges simply supported is maximum, basic frequency corresponding to two opposite edges simply supported and two opposite edges clamped is middle, and basic frequency corresponding to four edges clamped is minimum, but with the increase of $\gamma$, the influence of baffle boundary conditions on the basic frequency of the coupling vibration is gradually weakened; when the baffle position is close to the liquid level, the frequency corresponding to three kinds of boundary conditions is basically equivalent. On the whole, the relationship between coupling vibration frequency and the ratio $\gamma$ is parabola.

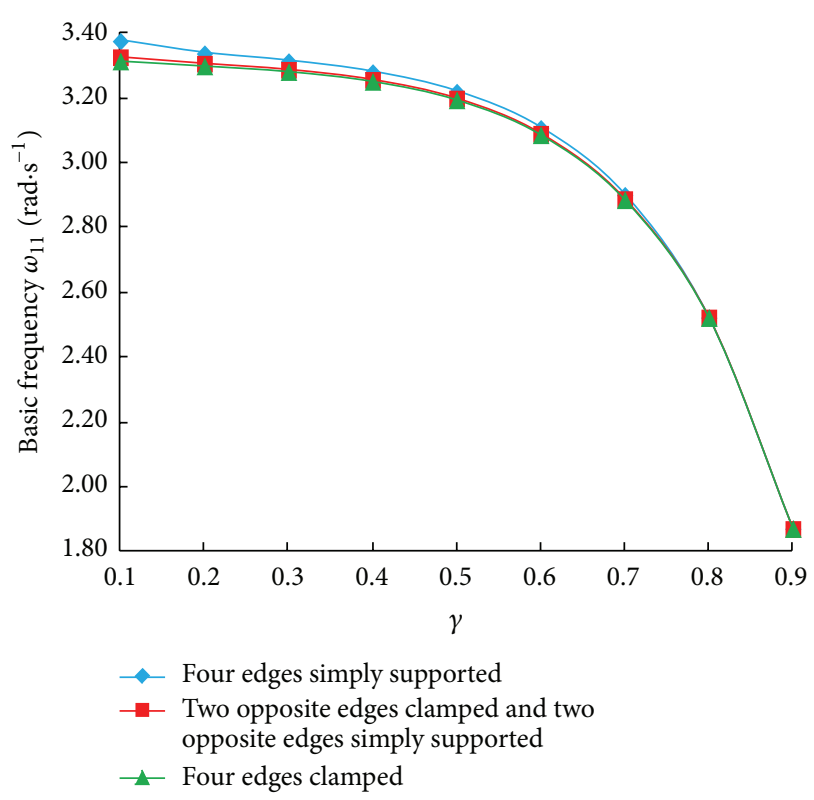

FIGURE 4: Effect of baffle height to liquid level ratio $\gamma$ on the basic frequency of liquid-solid coupling vibration.

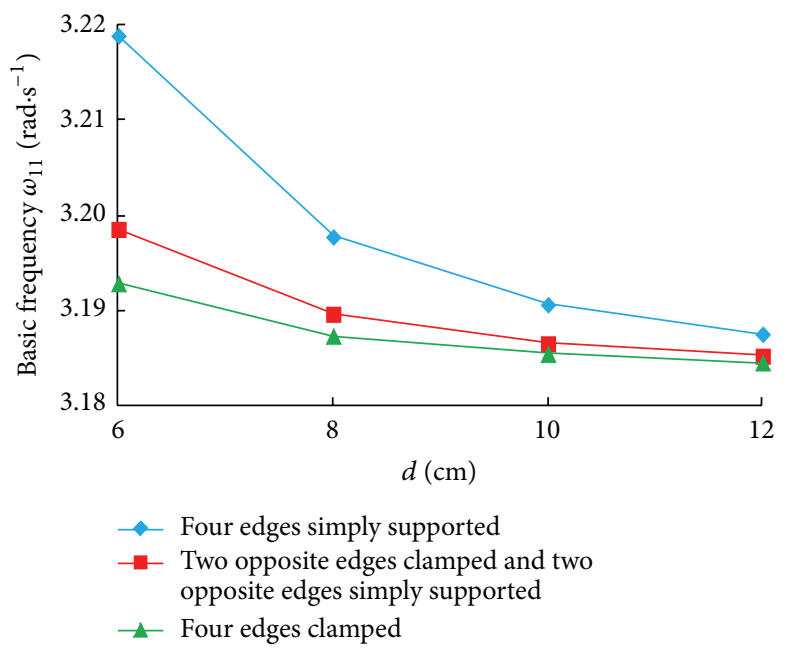

FIGURE 5: Effect of baffle thickness on the basic frequency of liquidsolid coupling vibration.

4.2.3. Effect of Baffle Thickness on the Basic Frequency of Liquid-Solid Coupling Vibration. The baffle thickness $d$ is, respectively, $6 \mathrm{~cm}, 8 \mathrm{~cm}, 10 \mathrm{~cm}$, and $12 \mathrm{~cm}$; in these cases, the relative baffle thickness $d / a$ is in the scope of elastic thin plate, the ratios $\lambda$ and $\gamma$ are 1.0 and $0.5, \rho$ is $2500 \mathrm{~kg} / \mathrm{m}^{3}, \rho_{w}$ is $1000 \mathrm{~kg} / \mathrm{m}^{3}, E$ is $30 \mathrm{GPa}$, and the influence of $d$ on the basic frequency of the coupling vibration is shown in Figure 5. As can be seen from Figure 5, the basic frequencies corresponding to the three boundary conditions all decrease with the increase of baffle thickness $d$, but with the increase of $d$, the trend that frequencies decrease becomes much gentler. When the baffle thickness is much thinner, the difference of the frequency corresponding to the three boundary conditions is 


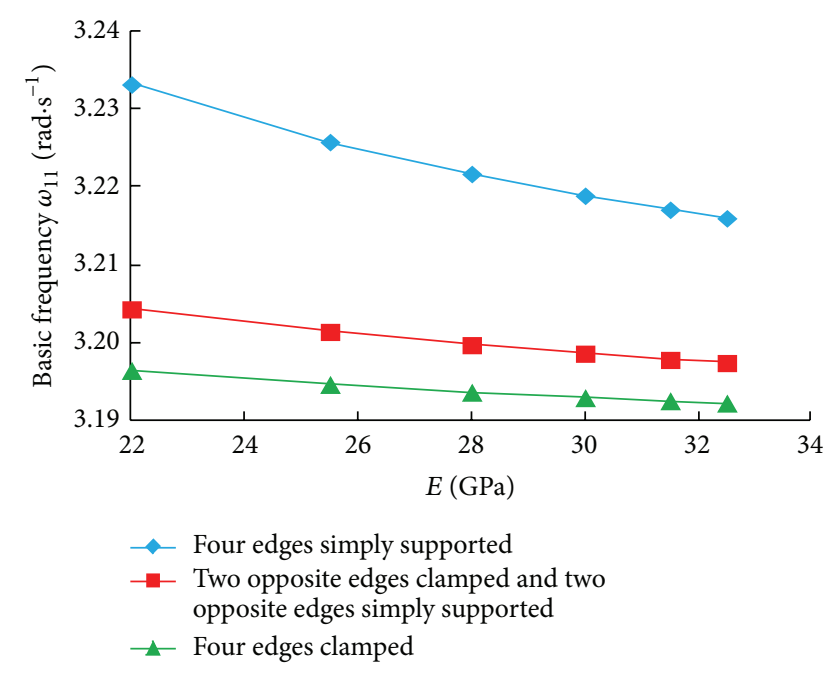

FIGURE 6: Effect of baffle modulus of elasticity on the basic frequency of liquid-solid coupling vibration.

relatively larger, but with the increase of baffle thickness, the influence of boundary conditions on the basic frequency of the coupling vibration is gradually weakened.

4.2.4. Effect of Baffle Elastic Modulus on the Basic Frequency of Liquid-Solid Coupling Vibration. The baffle elastic modulus $E$ is, respectively, $22 \mathrm{GPa}, 25.5 \mathrm{GPa}, 28 \mathrm{GPa}, 30 \mathrm{GPa}, 31.5 \mathrm{GPa}$, and $32.5 \mathrm{GPa}, \rho$ is $2500 \mathrm{~kg} / \mathrm{m}^{3}, d$ is $6 \mathrm{~cm}$, the ratios $\lambda$ and $\gamma$ are 1.0 and $0.5, \rho_{w}$ is $1000 \mathrm{~kg} / \mathrm{m}^{3}$, and the influence of $E$ on the basic frequency of the coupling vibration is shown in Figure 6. The results show that when the elastic modulus $E$ changes in the process of small to large value, the frequency of simply supported edges is obviously larger than that of the other two kinds of boundary conditions, and the basic vibration frequency decreases with the increase of elastic modulus, so in the design of reinforced concrete rectangular liquidstorage structure, in order to reduce the coupling vibration frequency, higher grade concrete can be used for baffle.

4.2.5. Effect of Baffle Density on the Basic Frequency of LiquidSolid Coupling Vibration. The baffle density $\rho$ is, respectively, $2100 \mathrm{~kg} / \mathrm{m}^{3}, 2200 \mathrm{~kg} / \mathrm{m}^{3}, 2300 \mathrm{~kg} / \mathrm{m}^{3}, 2400 \mathrm{~kg} / \mathrm{m}^{3}$, $2500 \mathrm{~kg} / \mathrm{m}^{3}$, and $2600 \mathrm{~kg} / \mathrm{m}^{3}, d$ is $6 \mathrm{~cm}$, the ratios $\lambda$ and $\gamma$ are 1.0 and $0.5, \rho_{w}$ is $1000 \mathrm{~kg} / \mathrm{m}^{3}, E$ is $30 \mathrm{GPa}$, and the influence of $\rho$ on the basic frequency of the coupling vibration is shown in Figure 7. As can be seen from Figure 7, the effect of baffle density on basic frequency of the coupling system is very small; therefore, the influence of baffle density on the basic frequency cannot be considered in the design.

4.2.6. Effect of Liquid Density on the Basic Frequency of LiquidSolid Coupling Vibration. The liquid density $\rho_{w}$ is, respectively, $800 \mathrm{~kg} / \mathrm{m}^{3}, 900 \mathrm{~kg} / \mathrm{m}^{3}, 1000 \mathrm{~kg} / \mathrm{m}^{3}$, and $1100 \mathrm{~kg} / \mathrm{m}^{3}, d$ is $6 \mathrm{~cm}$, the ratios $\lambda$ and $\gamma$ are 1.0 and $0.5, \rho$ is $2500 \mathrm{~kg} / \mathrm{m}^{3}$, $E$ is $30 \mathrm{GPa}$, and the influence of $\rho_{w}$ on the basic frequency of the coupling vibration is shown in Figure 8. As can be

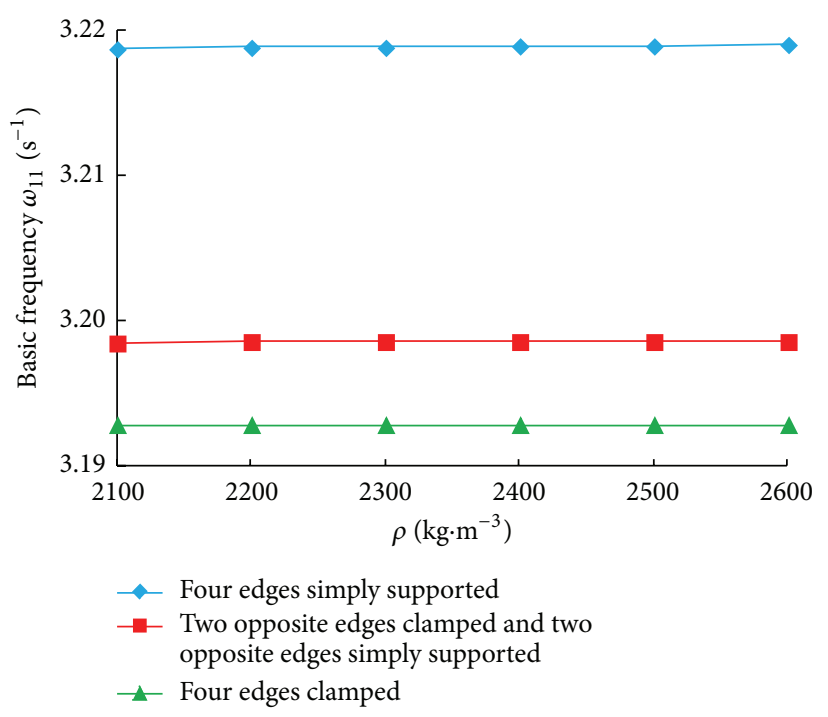

FIGURE 7: Effect of baffle density on the basic frequency of liquidsolid coupling vibration.

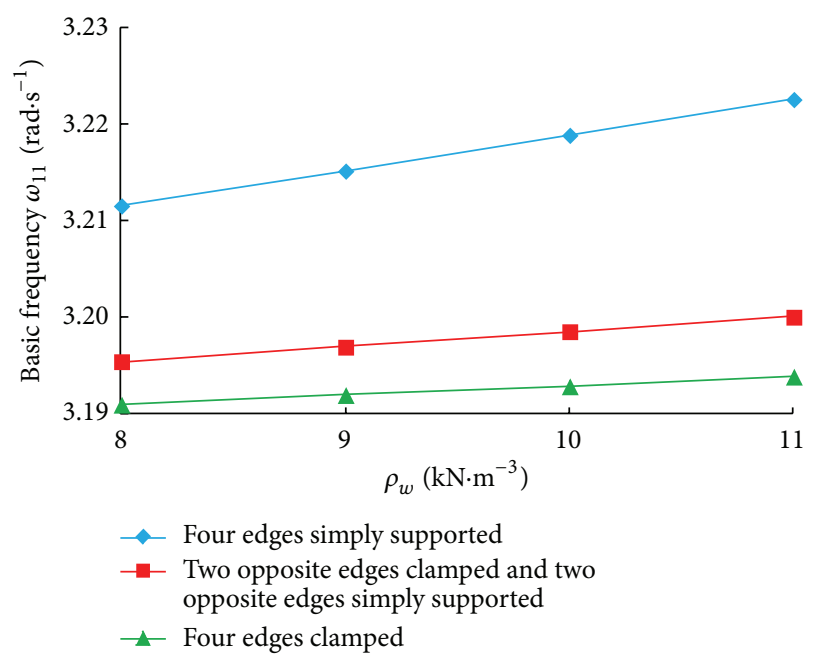

FIGURE 8: Effect of liquid density on the basic frequency of liquidsolid coupling vibration.

seen from Figure 8, the basic frequencies of the coupling vibration corresponding to the three boundary conditions all increase slowly with the increase of liquid density. Under various liquid densities, the frequency of simply supported edges is larger than that of the other two kinds of boundary conditions; compared with other parameters of the system, liquid density is positively correlated with basic frequency, but in the actual engineering, the difference of liquid density is small in general, so the effect of liquid density on the basic frequency also cannot be considered.

4.3. Solution of Coupled Modes. The results gotten from Section 4.2 show that the coupling vibration frequencies are affected by the parameters $\lambda$ and $\gamma$ significantly more than the other system parameters; due to limited space, the modes 


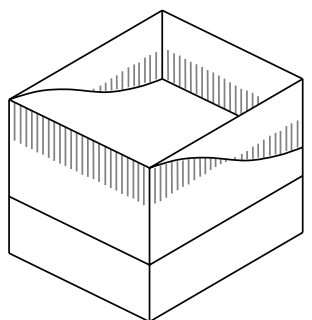

$\lambda=1.2$

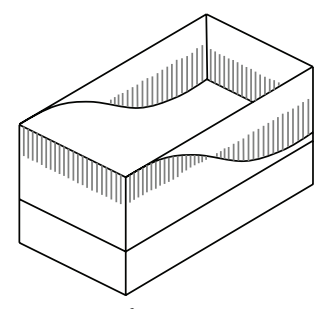

$\lambda=2.0$

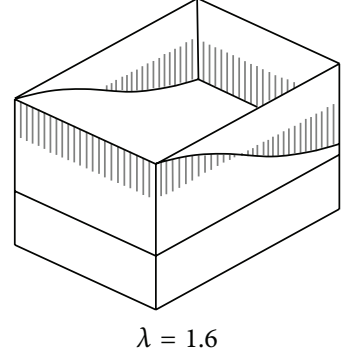

(a)

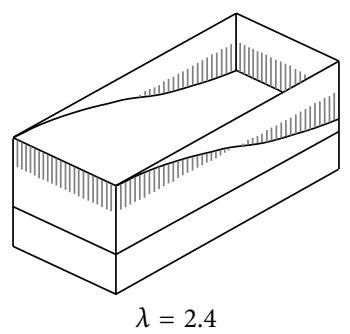

(b)

FIGURE 9: Coupled modes corresponding to $\lambda$.
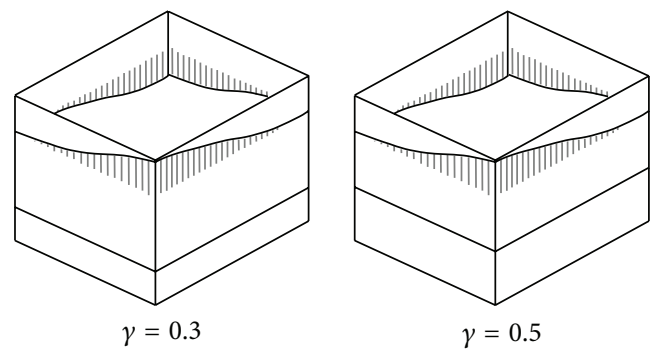

(a)
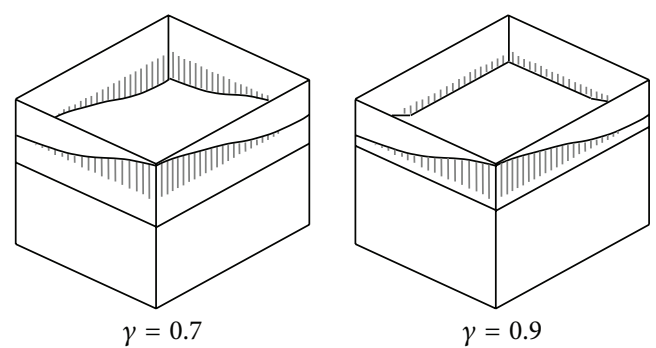

(b)

Figure 10: Coupled modes corresponding to $\gamma$.

corresponding to $\lambda(1.2,1.6,2.0,2.4)$ and $\gamma(0.5,0.5,0.7,0.9)$ are listed in Figures 9 and 10.

\section{Conclusions}

(1) The influence of baffle boundary conditions on the basic frequency of the coupling vibration of rectangular liquidstorage structure cannot be ignored. In general, under the same conditions, the basic frequency of the coupling vibration corresponding to four edges of baffle simply supported is maximum, frequency corresponding to two opposite edges simply supported and two opposite edges clamped is middle, and frequency corresponding to four edges clamped is minimum. Therefore, horizontal baffle should be connected to tank wall with four clamped edges when conducting sloshing reduction design; it not only is convenient for construction, but also can reduce the basic frequency of fluid-solid coupling vibration.

(2) Many of system parameters have a negative relation with the basic frequency, such as baffle elastic modulus, baffle thickness, baffle length-width ratio, and the ratio of baffle height to liquid level, while the liquid density is positively correlated with the basic frequency of the coupling vibration, but in the actual engineering, the difference of liquid density is small in general, so the effect of liquid density on the basic frequency of the coupling vibration cannot be considered. Besides, the effect of baffle density on the coupling frequency is very small and its effect can also be neglected.

(3) In contrast, the influences of length-width ratio and baffle height relative to liquid level on the basic frequency of the coupling vibration are much greater than that of the other system parameters; the larger the baffle length-width ratio, the closer the position of horizontal baffle to the liquid level; the coupling frequency will be reduced more obviously, and the relation between baffle length-width ratio and the coupling vibration frequency is exponential, while the ratio of baffle height to liquid lever has a parabola relation with the coupling vibration frequency.

\section{Competing Interests}

The authors declare that there are no competing interests regarding the publication of this paper.

\section{Acknowledgments}

This paper is a part of the support of the National Natural Science Foundation of China (Grant nos. 51368039, 51478212), a part of the support of the Education Ministry Doctoral Tutor Foundation of China (Grant no. 20136201110003), a part of the support of the Plan Project of Science and Technology in Gansu Province (Grant no. 144GKCA032), and a part of the support of the National Key Basic Research and Development Plan of China (973 Plan, Grant no. 2011CB013600).

\section{References}

[1] X. D. Cheng, "Equivalent mechanical model of liquid sloshing in a cylindrical tank with elastic plate," Journal of Anqing Teachers College (Natural Science Edition), vol. 4, no. 1, pp. 3740, 1998.

[2] Z. L. Wang, X. D. Cheng, and B. Quan, "Liquid sloshing in spherical tank with space at low gravity environment," Chinese Journal of space Science, vol. 10, no. 2, pp. 107-119, 1990.

[3] B. Z. Yue, Z. L. Wang, J. F. Li, and J. L. Kuang, "Liquid sloshing in cylindrical tank with elastic spacer," Journal of Tsinghua University (Science and Technology), vol. 37, no. 8, pp. 26-28, 1997.

[4] M. Yang, J. F. Li, T. S. Wang, and W. Wang, "Determination of damping ratio of the small-amplitude liquid sloshing within 
the cylindrical container with a ring baffle," Chinese Journal of Theoretical and Applied Mechanics, vol. 38, no. 5, pp. 660-667, 2006.

[5] Y. J. Hao, Y. Yang, and X. Z. Bai, "Analysis of deformation and stress of elastic plate in a tank under the influence of fluid," Journal of Mechanical Strength, vol. 31, no. 2, pp. 250-255, 2009.

[6] K. C. Biswal and S. K. Bhattacharyya, "Dynamic response of structure coupled with liquid sloshing in a laminated composite cylindrical tank with baffle," Finite Elements in Analysis and Design, vol. 46, no. 11, pp. 966-981, 2010.

[7] M. Shahrokhi, F. Rostami, M. A. M. Said, and Syafalni, "Numerical modeling of baffle location effects on the flow pattern of primary sedimentation tanks," Applied Mathematical Modelling, vol. 37, no. 6, pp. 4486-4496, 2013.

[8] J. D. Wang, D. Zhou, and W. Q. Liu, "Study of coupling vibration characteristics of a cylindrical tank with a flexible annual baffle," Engineering Mechanics, vol. 29, no. 6, pp. 270-276, 2012.

[9] W.-H. Song, D.-Z. Ning, Y.-L. Liu, and T. Bing, "Numerical simulation of liquid sloshing in a container with baffles," Chinese Journal of Hydrodynamics, vol. 27, no. 1, pp. 54-61, 2012.

[10] M.-A. Xue, J. H. Zheng, and P. Lin, "Numerical simulation of sloshing phenomena in cubic tank with multiple baffles," Journal of Applied Mathematics, vol. 2012, Article ID 245702, 21 pages, 2012.

[11] J. D. Wang, D. Zhou, and W. Q. Liu, "Study on coupled vibration characteristics of a cylindrical container with multiple elastic annular baffles," Science China Technological Sciences, vol. 55, no. 12, pp. 3292-3301, 2012.

[12] S. M. Hasheminejad, M. M. Mohammadi, and M. Jarrahi, "Liquid sloshing in partly-filled laterally-excited circular tanks equipped with baffles," Journal of Fluids and Structures, vol. 44, pp. 97-114, 2014.

[13] M. R. Shekari, "Seismic response of liquid-filled tank with baffles," Journal of Marine Science and Application, vol. 13, no. 3, pp. 299-304, 2014.

[14] M. Ebrahimian, M. A. Noorian, and H. Haddadpour, "Free vibration sloshing analysis in axisymmetric baffled containers under low-gravity condition," Microgravity Science and Technology, vol. 27, no. 2, pp. 97-106, 2015.

[15] M. A. Goudarzi and P. Farshadmanesh, "Numerical evaluation of hydrodynamic damping due to the upper mounted baffles in real scale tanks," Soil Dynamics and Earthquake Engineering, vol. 77, pp. 290-298, 2015.

[16] X.-S. Cheng and Y.-F. Du, "The dynamic fluid pressure of reinforced concrete rectangular liquid-storage tanks with elastic walls," Engineering Mechanics, vol. 26, no. 6, pp. 82-88, 2009.

[17] Z. L. Wang and B. Quan, "Sloshing of liquid in the spherical tank with spaces of netted-holes," Chinese Journal of space Science, vol. 9, no. 2, pp. 136-147, 1989.

[18] X. D. Cheng and Z. L. Wang, "Hydroelastic vibrations in rectangular tank with elastic spacer," Mechanics in Engineering, vol. 21, pp. 50-52, 1999.

[19] N. R. Mo, Engineering Fluid Mechanics, Huazhong University of Science and Technology Press, Wuhan, China, 2003 (Chinese).

[20] T. Y. Tsui and N. C. Small, "Hydroelastic oscillations of a liquid surface in an annular circular cylindrical tank with flexible bottom," Journal of Spacecraft \& Rockets, vol. 2, no. 2, pp. 202206, 1968.

[21] X. S. Cheng and Y. F. Du, "Liquid-solid coupling sloshing of reinforced concrete rectangular liquid-storage tanks-elastic soleplate," Chinese Journal of Applied Mechanics, vol. 25, no. 4, pp. 622-626, 2008.
[22] S. L. Ding and W. G. Bao, "Modal analysis and equivalent mechanical model of liquid sloshing in arbitrary 3D container," Chinese Quarterly of Mechanics, vol. 25, no. 1, pp. 62-68, 2004.

[23] Z. J. Zou, "Horizontal aseismatic calculation of a multistory structure with large sewage pools," Chinese Quarterly of Mechanics, vol. 24, no. 1, pp. 120-123, 2003. 


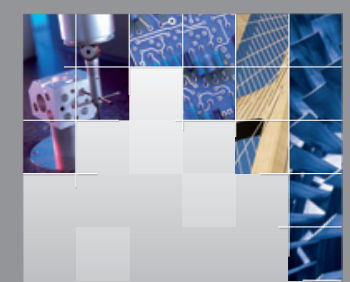

\section{Enfincering}
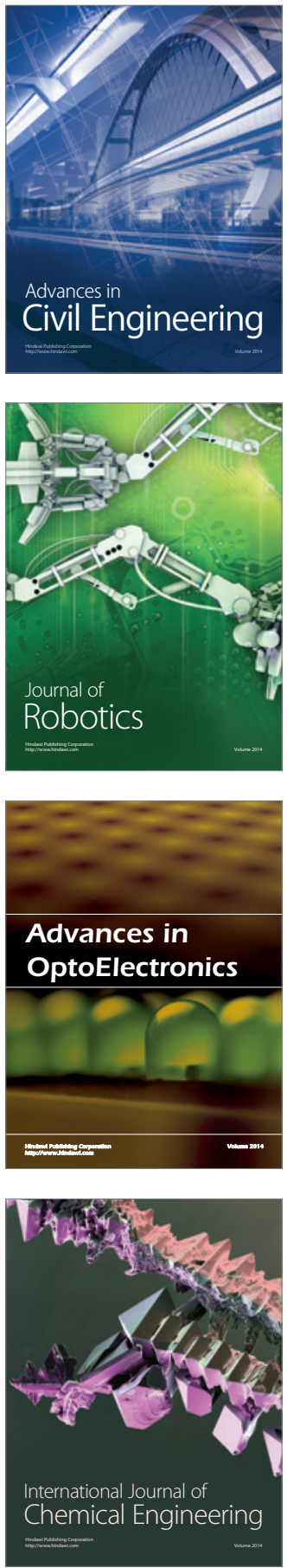

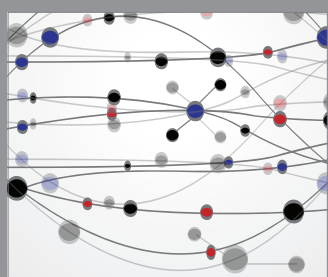

The Scientific World Journal

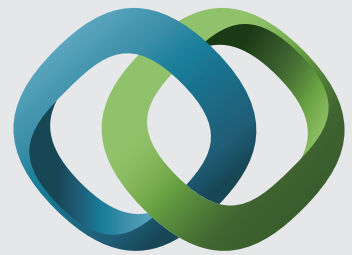

\section{Hindawi}

Submit your manuscripts at

http://www.hindawi.com
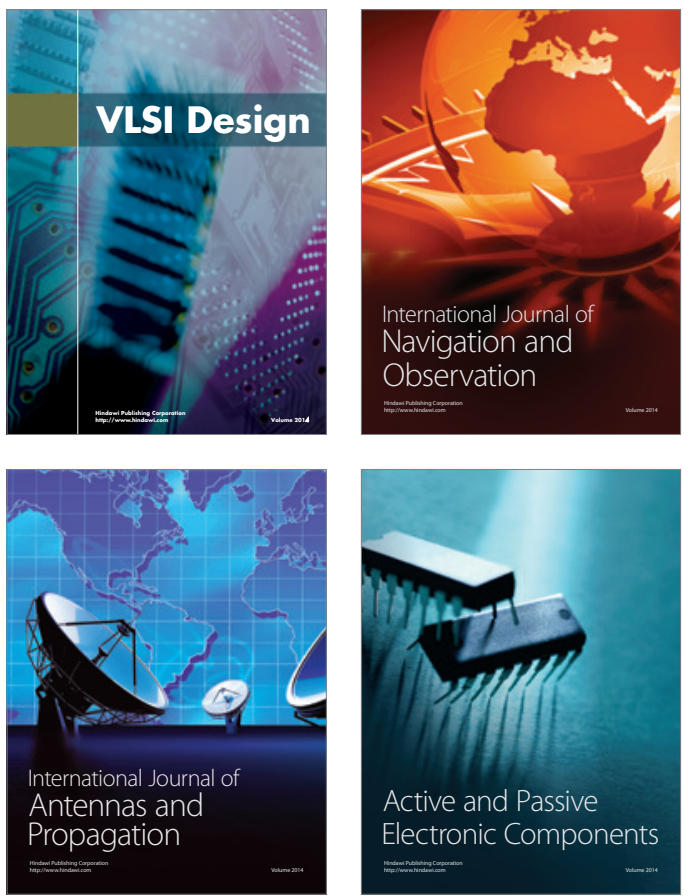
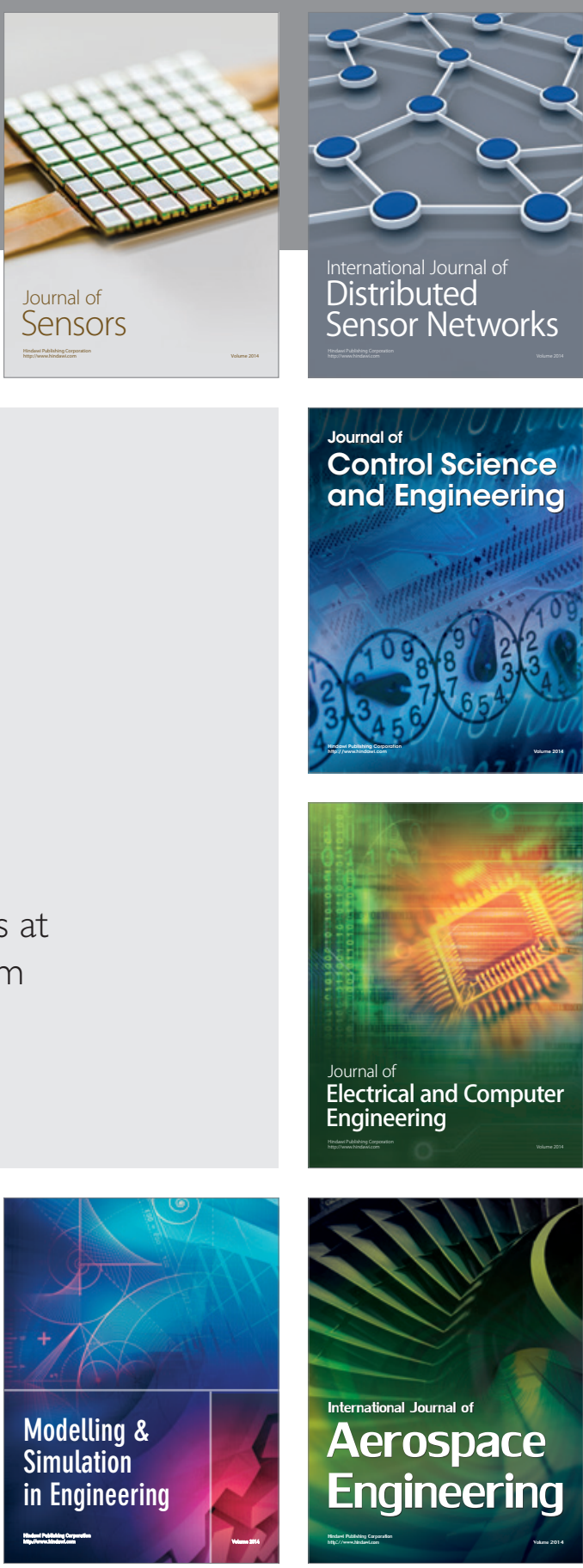

International Journal of

Distributed

Sensor Networks

Journal of

Control Science

and Engineering
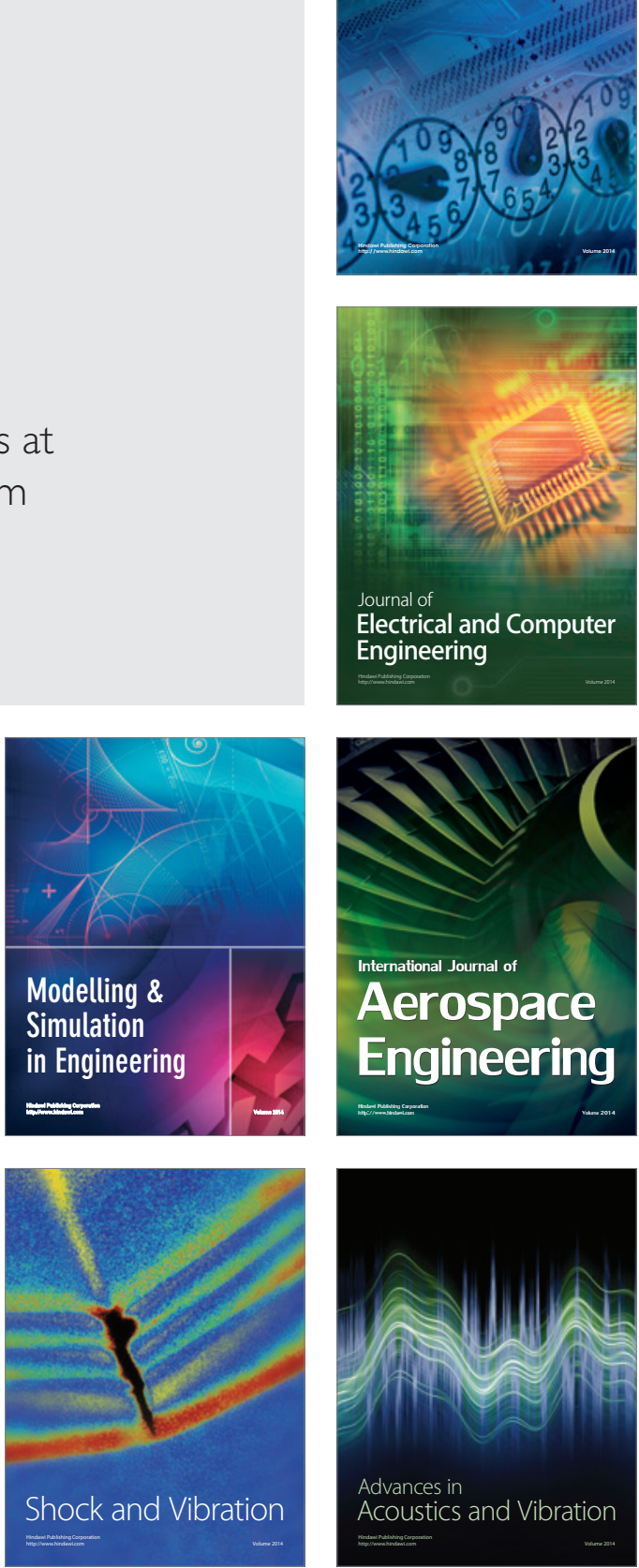